\title{
Caudate Dopamine D1 Receptor Density Is Associated with Individual Differences in Frontoparietal Connectivity during Working Memory
}

\author{
Anna Rieckmann, ${ }^{1}$ Sari Karlsson, ${ }^{1}$ Håkan Fischer, ${ }^{2}$ and Lars Bäckman ${ }^{1}$ \\ ${ }^{1}$ Aging Research Center, Karolinska Institute, E-113 30 Stockholm, Sweden, and ${ }^{2}$ Department of Psychology, Stockholm University, E-106 91 Stockholm, \\ Sweden
}

We assess the relationship of age-related losses in striatal D1 receptor densities to age-related reductions in functional connectivity between spatially distinct cortical regions in healthy human participants. Previous neuroimaging studies have reported age-related differences in functional connectivity of the frontoparietal working memory network and the default mode network during task performance. We used functional magnetic resonance imaging and seed-based connectivity (right dorsolateral and medial prefrontal cortex) to extend these findings: Anterior-posterior connectivity of both these functional networks was reduced in older (65-75 years, $n=18$ ) compared with younger (20-30 years, $n=19)$ adults, whereas bilateral connectivity in prefrontal cortex was increased in older adults. Positron emission tomography with the $\mathrm{D} 1$ receptor ligand $\left[{ }^{11} \mathrm{C}\right] \mathrm{SCH} 23390$ was used to assess caudate D1 receptor density in the same sample. Older adults showed significantly reduced caudate D1 receptor density compared to the younger adults. Of key interest, partial correlations showed that individual differences in caudate D1 receptor density were positively associated with individual differences in dorsolateral prefrontal connectivity to right parietal cortex (BA40) and negatively with medial prefrontal connectivity to right parietal cortex (BA40 and postcentral gyrus), after controlling for age. We found no correlation of caudate D1 receptor density with anteriorposterior coupling within the default mode network or with bilateral frontal connectivity. These results are consistent with animal work that has identified a role for caudate D1 receptors in mediating information transfer between prefrontal areas and parietal cortex.

\section{Introduction}

Functional magnetic resonance imaging (fMRI) studies have shown that healthy younger adults typically engage a frontoparietal network during working memory (WM). The frontoparietal WM network broadly includes the dorsolateral prefrontal cortex (DLPFC), supplementary motor areas, and parietal areas (BA 7/40), whereas medial prefrontal cortex (MPFC), lateral (posterior) parietal areas (BA 39), and posterior cingulate (PCC) are not involved (Fox et al., 2005; Toro et al., 2008). By contrast, the latter areas are part of the default mode network (DMN), which is engaged during resting state (Buckner et al., 2008). It has been suggested that the task-positive frontoparietal network and the task-negative DMN work antagonistically (Fox et al., 2005), and the degree of "anticorrelation" has been linked to better WM functioning (Hampson et al., 2010).

\footnotetext{
Received June 19, 2011; revised July 22, 2011; accepted Aug. 9, 2011.

Author contributions: S.K., H.F., and L.B. designed research; S.K., H.F., and L.B. performed research; A.R. and S.K. analyzed data; A.R. wrote the paper.

This work was supported by grants from the Swedish Research Council to L.B., from Swedish Brain Power, an Alexander von Humboldt Research Award, and a donation from the af Jochnick Foundation to L.B. We are grateful to Per Karlsson, Lars Farde, and Zsolt Cselenyi for their input on the PET data collection and analysis, and to Martin Lövden and Lars Nyberg for comments on an earlier version of the paper.

The authors declare no competing financial interests.

Correspondence should be addressed to Anna Rieckmann, Aging Research Center, Karolinska Institute, Gävlega$\tan 16$, S-113 30 Stockholm, Sweden. E-mail: anna.rieckmann@ki.se.

DOI:10.1523/JNEUROSCI.3114-11.2011

Copyright $\odot 2011$ the authors $\quad 0270-6474 / 11 / 3114284-07 \$ 15.00 / 0$
}

Age-related decreases in WM are well documented (for review, see Bäckman et al., 2001), and recent studies have linked age-related cognitive deficits to changes in frontoparietal and DMN connectivity during task performance. These studies suggest that old age is associated with reduced connectivity between MPFC and PCC in the DMN (Andrews-Hanna et al., 2007; Grady et al., 2010; Sambataro et al., 2010), reduced frontoparietal connectivity in the task-positive network (Andrews-Hanna et al., 2007; but see Grady et al., 2010), particularly during demanding task conditions (Nagel et al., 2011), and reduced negative connectivity between PCC and DLPFC (Sambataro et al., 2010). Thus, age-related changes in functional connectivity appear most pronounced in anterior-posterior coupling, both within and between the frontoparietal network and the DMN. Interestingly, Grady et al. (2010) also found that older adults showed a pattern of increased connectivity within frontal areas.

An emerging hypothesis is that age-related decreases in dopamine (DA) functions (Bäckman et al., 2006, 2010) may be related to altered cortical connectivity in aging. Striatal DA receptors modulate cortico-striato-cortical connections by which information is relayed between cortical areas (Joel and Weiner, 2000). Moreover, pharmacological DA challenges (Nagano-Saito et al., 2009) and positron emission tomography (PET) studies in younger healthy adults (Braskie et al., 2011) have linked the striatal DA system to DMN deactivation during cognitive task performance. Finally, several studies have shown a link between striatal DA measured by PET (e.g., Schott et al., 2008; Landau et 
al., 2009; Bäckman et al., 2011) or SPECT (Nyberg et al., 2009) and $\mathrm{fMRI}$ activations. The precise mechanisms that link striatal DA and blood oxygen level-dependent (BOLD) signal are not fully understood, but it has been suggested that the link between striatal DA and BOLD response is primarily mediated by postsynaptic DA D1 receptor activity (Knutson and Gibbs, 2007).

Collectively, these studies suggest a link between striatal DA activity and cortical connectivity, but empirical support for the hypothesis that DA functions are linked to age-related connectivity changes is still lacking. The present study examined the association between caudate DA D1 receptor density and functional connectivity during WM in younger and older adults. We hypothesized an association between individual differences in D1 receptor density and individual differences in functional connectivity independent of chronological age.

\section{Materials and Methods}

\section{Participants}

Participants were 19 healthy younger ( 9 women; mean age $=25.16, \mathrm{SD}=$ 2.27) and 18 older ( 9 women; mean age $=70.33, \mathrm{SD}=3.25$ ) adults, who were recruited by newspaper advert. There was no difference in years of education between groups $\left(\right.$ mean $_{\text {younger }}=14.61, \mathrm{SD}=2.00$; mean $_{\text {older }}=$ $14.72, \mathrm{SD}=3.96 ; t<1$ ), and all participants were right handed, nonsmokers, and free from past or present drug or alcohol abuse, neuropsychiatric disorders, or brain damage. No woman was on hormone replacement therapy. The study was approved by the Ethics and Radiation Safety Committees at the Karolinska Hospital, Stockholm, Sweden.

\section{Procedure}

All participants took part in a PET and an MRI session, lasting $\sim 1 \mathrm{~h}$ each, and not separated by more than 2 months. Participants were paid for their participation. During the PET measurement, participants were instructed to rest. The instructions and design for the MRI session are detailed below.

\section{Working memory task}

During the fMRI acquisition, participants performed a spatial WM task, which has been described in detail previously (Fischer et al., 2010; Bäckman et al., 2011). Briefly, participants were presented with a $4 \times 4$ grid at the center of the computer screen. For each WM trial, either 4 (low load) or 6 (high load) target circles consecutively lit up in random positions of the grid for $900 \mathrm{~ms}$. After a delay of $2 \mathrm{~s}$, a probe circle lit up and participants were to indicate by button press whether any of the target circles had appeared in the position of the probe circle. In total, participants were presented with 15 high-load and 15 low-load trials ( 3 per block), and accuracy and reaction times (RTs) of correct responses were recorded as a measure of WM performance and used as the dependent variables in a 2 (age) $\times 2$ (load) ANOVA. A perceptually matched baseline condition was alternated with WM blocks.

\section{MRI data}

All images were acquired on a 1.5 tesla MRI system (Signa Excite HD Twinspeed, General Electrics Medical Systems). Participants viewed stimuli from a projector (Philips Hopper HG 20 Impact LCD projector) via a coil mirror, and responded via an fMRI-compatible button set using the right index and middle finger (Psychology Software Tools).

Acquisition. A T1-weighted 3D-SPGR sequence $(\mathrm{TR}=24 \mathrm{~ms}, \mathrm{TE}=6$ $\mathrm{ms}$, flip angle $=35^{\circ}$, slice thickness $1.5 \mathrm{~mm}$, FOV $256 \mathrm{~mm}$ ) was used for coregistration with the functional MRI and PET images. Functional data were collected using an EPI sequence $(\mathrm{TR}=2.5 \mathrm{~s}$, TE $=40 \mathrm{~ms}$, flip angle $=90^{\circ}$, slice thickness $=4 \mathrm{~mm}$ with $0.5 \mathrm{~mm}$ gap, interleaved, FOV $220 \mathrm{~mm}$ ). Two EPI runs of 140 volumes each were entered into the fMRI analyses, after removal of the first four volumes as dummy scans.

Analysis. All analyses were performed using FEAT v.5.98 as part of FSL 4.1.6 (FMRIB Software Library) (Smith et al., 2004). Functional data were motion corrected using rigid-body transformations (MCFLIRT, Jenkinson et al., 2002), masked for nonbrain tissue using FSL's BET brain extraction tool, and smoothed (FWHM kernel $8 \mathrm{~mm}$ ). Grand-mean scal- ing was applied to the whole dataset and a high-pass filter was used to remove low-frequency signals. Next, whole-brain connectivity maps were produced using General Linear Modeling (GLM) with two initiating seeds (cf. Andrews-Hanna et al., 2007; Sambataro et al., 2010; Nagel et al., 2011). Seeds were one task-positive prefrontal area (right DLPFC; MNI coordinates $x=36, y=36, z=26$ ) and one task-negative prefrontal area (MPFC; $x=-2, y=38, z=-6$ ) based on the task-based contrasts, $\mathrm{WM}>$ Baseline and Baseline $>\mathrm{WM}$, respectively. We used right DLPFC and MPFC as initiating seeds because of their strong involvement in the frontoparietal network and the DMN, respectively, and the relevance of the (right) prefrontal cortex to spatial WM (e.g., Callicott et al., 1999; Baddeley, 2003; Curtis and D'Esposito, 2003). FLIRT (FMRIB linear image registration tool, Jenkinson et al., 2002) was used to register the seeds to functional space before time series extraction. To ensure an unbiased seed selection, we identified seeds as peak activations (with a 10 $\mathrm{mm}$ ROI) in an independent sample of 46 younger and older adults that performed a task identical to that used in the present study.

The model included the time series of the seed, a task-based regressor (modeling low and high WM load as -1 and 1, respectively, and convolved with a double-gamma hemodynamic response function as a model of the hemodynamic response of the physiological regressor), and an interaction term between the time series regressor (centered at zero) and the task regressor to identify a psychophysiological interaction (PPI). The PPI was included as a regressor because one previous study has identified age-related differences in load-related functional connectivity during WM task performance (Nagel et al., 2011). The GLM also included nuisance regressors (white matter time series, CSF time series, global signal time series, and 6 motion parameters) to account for artifactual signals. The functional data were coregistered to a standard brain template (MNI152) via the structural images. Whole-brain first-level analyses of the two runs were averaged across runs using fixed effects and then averaged within age groups in a higher-level random-effects analysis, with a voxelwise significance threshold of $p<0.01$. Correction for multiple comparisons was applied at the cluster level using Gaussian Random Field theory (GRF; $p<0.05$ ). This analysis yielded mean functional connectivity maps for each age group based on the time series regressor as well as mean PPI maps for each age group. Voxels with maximally significant age group differences were masked with a $6 \mathrm{~mm}$ cubic ROI to extract individual mean parameter estimates (converted to percentage signal change using FSL's Featquery) as (1) a measure of functional connectivity strength of that area with the respective seed region (for the time series regressor) or (2) a measure of PPI of that area with the respective seed. These values were then cleaned for outliers $(>3$ SD) and used in further regression analyses with the PET data using SPSS v.18.0.

\section{PET data}

Acquisition. Dynamic PET data were obtained with an ECAT Exact HR 47 system (CTI/Siemens) run in 3D mode and with a transaxial resolution of $3.8 \mathrm{~mm}$ FWHM at the central field of view and $4.5 \mathrm{~mm}$ radially at 20 $\mathrm{cm}$ from the center. Emission data were recorded over a period of $51 \mathrm{~min}$ in 13 time frames after a transmission measurement of 10 min with 3 rotating 68Ge/68Ga sources. $\left[{ }^{11} \mathrm{C}\right] \mathrm{SCH} 23390$ (300 MBq) (Halldin et al., 1986) was injected into the left antecubital vein. A plaster helmet was made for each participant individually and used to fixate the head and minimize motion. Light was dimmed and auditory stimulation was kept to a minimum during acquisition of the PET data.

Analysis. The bilateral caudate nuclei were manually delineated on the structural MRI scans for each individual using Human Brain Atlas software (Roland et al., 1994). The cerebellum, where DA D1 receptor density is negligible, was delineated on six central slices and served as reference region (Hall et al., 1988). All PET analyses were performed using SPM5 (http://www.fil.ion.ucl.ac.uk/spm/) and in-house scripts implemented through MATLAB R2007b. MRI images were segmented into gray matter, white matter, and CSF, and coregistered with the PET images using SPM's mutual information coregistration. Gray matter MRI ROIs were used to derive time activity curves (regional radioactivity was calculated for each frame, corrected for decay, and plotted vs time). D1 receptor density was measured as the binding potential (BP) of 
$\left[{ }^{11} \mathrm{C}\right] \mathrm{SCH} 23390$, pooled across hemispheres. Here, BP is the ratio at equilibrium of specifically bound radioligand to that of nondisplaceable radioligand in tissue (Innis et al., 2007) and calculated using the simplified reference tissue model, with the cerebellum as reference region (Lammertsma and Hume, 1996). PET data were corrected for partialvolume effects (Meltzer et al., 1990). The PET analysis was restricted to the caudate, because previous work has identified structural connectivity of the caudate, but not the putamen, with the DMN and frontoparietal association areas (e.g., Lehéricy et al., 2004; Grahn et al., 2008; Ystad et al., 2011). Relatedly, past studies that have combined DA PET and fMRI have reported a selective role for caudate DA functioning in WM performance and WM-related fMRI activations (Landau et al., 2009; Wallace et al., 2011).

\section{Linking PET and $f M R I$}

For all ROIs where we identified age-related differences in functional connectivity (from the time series regressor) or in the PPI maps (from the PPI regressor) in the fMRI analysis, we explored the association between D1 receptor density in the caudate and individual differences in functional connectivity or PPI, respectively. We computed partial correlations between D1 receptor density and fMRI parameter estimates (converted to percentage signal change) after controlling for chronological age. Where we found a significant association between D1 receptor density and fMRI parameter estimates, we also used linear regressions to check for significant age $\times$ D1 interactions. An absence of a significant interaction indicates that the associations between D1 and functional connectivity generalize across the two age groups.

\section{Results}

\section{Behavioral}

For both accuracy and RT, there was a main effect of age (Accuracy: $F_{(1,35)}=45.63, p<0.001$; RT: $\left.F_{(1,35)}=5.33, p<0.03\right)$, but no main effect or interaction with load ( $p s>0.14)$. Across load conditions, younger adults had a mean accuracy of $95.93 \%$ (mean number correct $=14.39 ; \mathrm{SD}=0.66)$ and a mean $\mathrm{RT}$ of $896.76 \mathrm{~ms}$ ( $\mathrm{SD}=119.72)$, and older adults had a mean accuracy of $75.93 \%$ (mean number correct $=11.39$; SD $=1.82$ ) and a mean RT of $1004.20 \mathrm{~ms}$ (SD = 161.29). There were no significant correlations of caudate D1 receptor densities to WM accuracy or RT across the whole sample or within age groups.

\section{Psychophysiological interaction}

There were no significant effects for the PPI regressor in younger adults, suggesting that the strength of functional connectivity with the seeds did not differ by task load. Older adults showed greater connectivity of the DLPFC seed to the paracingulate and bilateral middle frontal gyri with higher task load, and this cluster was also the only one to survive statistical comparison between age groups (medial frontal: $x=8, y=30, z=38$; right middle frontal: $x=18, y=42, z=32$; left middle frontal: $x=-24, y=$ $34, z=30$ ). There were no significant associations between (1) PPI parameter estimates and WM accuracy or RT and (2) PPI parameter estimates and D1 receptor density across the sample, after controlling for age. Because we found no PPI effects in the young, and no relations to performance or D1 receptor density, we will not discuss the PPI results further, but rather focus on the functional connectivity estimates derived from the time series regressor.

\section{Functional connectivity}

The mean functional connectivity maps that were generated based on the time series regressor of the two prefrontal seeds are shown in Figure 1 by age group. Using right DLPFC as the initiating seed in younger adults revealed positive connectivity in a frontoparietal WM network spanning the bilateral DLPFC, ante-
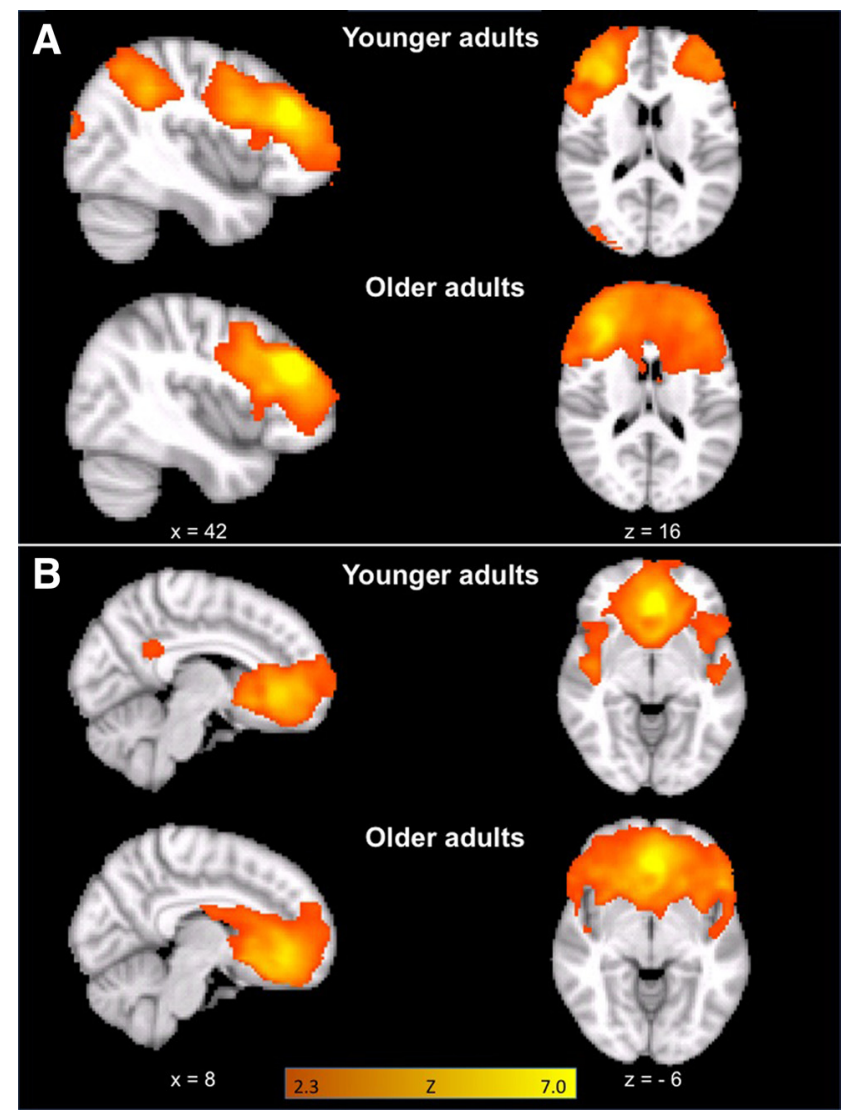

Figure 1. Mean connectivity maps for the right DLPFC seed $(\boldsymbol{A})$ and the MPFC seed $(\boldsymbol{B})$ by age group. $x$ and $z$ refer to coordinates in MNI standard space. The color bar indicates the range of the Z-statistic.

rior cingulate, anterior insula, and precentral cortices, as well as bilateral parietal cortices (BA7/40), precuneus, and occipital cortex. Negative connectivity of the DLPFC seed was found with the MPFC, PCC, bilateral posterior insula, bilateral middle temporal gyri and hippocampi, and cerebellum, which maps onto previous reports on the DMN (e.g., Buckner et al., 2008). For older adults, positive connectivity of the DLPFC seed was seen with the contralateral DLPFC, anterior cingulate, and MPFC, and negative connectivity to the PCC and cerebellum. Interestingly, we observed a significant negative correlation $(r=-0.50, p<0.01)$ between right DLPFC connectivity to right inferior parietal cortex (BA40) and right DLPFC connectivity to the left inferior frontal gyrus (BA 46) across the whole sample, indicating that greater connectivity of the DLPFC with the contralateral prefrontal cortex was related to decreased connectivity of the DLPFC to right parietal cortex. This correlation remained significant after partialing out age $(r=-0.33, p=0.05)$.

As expected based on the idea that DLPFC and MPFC work antagonistically, the reverse pattern of activations was found for the MPFC seed: Younger adults showed positive connectivity of the MPFC to PCC, as well as posterior insula, and negative connectivity to the DLPFC, precentral and parietal cortices, and the occipital lobe. For older adults, positive connectivity of the MPFC extended to orbitofrontal cortices and the anterior striatum, whereas negative connectivity was confined to precentral areas and the occipital lobe.

\section{Age differences in functional connectivity}

A detailed list of local peak age differences and corresponding mean signal changes in younger and older adults is shown in 
Table 1. Age-related differences in functional connectivity

\begin{tabular}{|c|c|c|c|c|c|c|c|}
\hline Seed & Regional peak age differences & $x$ & $y$ & $z$ & $Z$ (Younger $>$ Older) & Mean psc Younger & Mean psc Older \\
\hline \multirow[t]{8}{*}{ Right DLPFC } & Right superior parietal cortex (BA 7) & 34 & -62 & 50 & 3.89 & $0.38(0.31)$ & $-0.12(0.44)$ \\
\hline & Right inferior parietal lobe $(\mathrm{BA} 40)^{+}$ & 60 & -30 & 34 & 3.04 & $0.35(0.32)$ & $-0.47(0.33)$ \\
\hline & Left superior precuneus & 10 & -70 & 52 & 3.87 & $0.58(0.38)$ & $-0.02(0.43)$ \\
\hline & Left inferior parietal lobe (BA 7/40) & -42 & -52 & 46 & 3.21 & $0.39(0.28)$ & $0.07(0.25)$ \\
\hline & MPFC & 2 & 64 & -10 & -3.77 & $-0.51(0.54)$ & $0.33(0.82)$ \\
\hline & Left inferior frontal gyrus (BA 47) & -46 & 26 & 8 & -3.75 & $-0.06(0.33)$ & $0.32(0.40)$ \\
\hline & Right occipitotemporal sulcus & 36 & -62 & -10 & 3.25 & $-0.03(0.27)$ & $-0.26(0.32)$ \\
\hline & Midbrain $^{+}$ & 8 & -28 & -10 & 3.09 & $0.13(0.49)$ & $-0.19(0.32)$ \\
\hline \multirow[t]{6}{*}{ MPFC } & $P C C^{*}$ & -14 & -56 & 14 & 3.42 & $0.17(0.19)$ & $-0.08(0.19)$ \\
\hline & Right intraparietal sulcus (BA 40) ${ }^{+}$ & 28 & -46 & 48 & -4.04 & $-0.26(0.23)$ & $-0.02(0.18)$ \\
\hline & Postcentral gyrus ${ }^{+}$ & 50 & -22 & 38 & -3.08 & $-0.20(0.15)$ & $-0.02(0.15)$ \\
\hline & Right inferior frontal gyrus & 56 & 10 & 16 & -3.42 & $-0.13(0.15)$ & $0.10(0.16)$ \\
\hline & Right prefrontal cortex (BA 46) & 46 & 48 & 12 & -3.29 & $-0.19(0.42)$ & $0.30(0.57)$ \\
\hline & Right anterior insula & 34 & 22 & 10 & -3.28 & $-0.08(0.22)$ & $0.21(0.24)$ \\
\hline
\end{tabular}

$x, y$, and $z$ refer to coordinates of the peak age differences in MNI space. Psc values show the mean (and SD) parameter estimates for each age group separately (converted to percentage signal change and based on a $6 \mathrm{~mm}{ }^{3} \mathrm{ROI}$ ) in order to illustrate the origin of the age effect (Z). ${ }^{*}$ Significant at $p<0.001$ (did not survive GRF cluster-size correction for multiple comparisons). ${ }^{+}$ROls for which we found significant associations with D1 receptor density (Fig. 2).

Table 1. For the right DLPFC seed, younger adults showed stronger connectivity with bilateral parietal cortices (BA 7 and 40), precuneus, and midbrain and stronger negative connectivity to MPFC. By contrast, older adults showed stronger connectivity of the right DLPFC seed to the left inferior frontal gyrus, cerebellum, and occipital cortex.

For the MPFC seed, younger adults displayed stronger negative connectivity to the right parietal cortex, including the intraparietal sulcus (BA 40) and the postcentral gyrus and right prefrontal areas (inferior frontal gyrus and BA 46), but increased connectivity of the MPFC to the PCC (although age differences in PCC failed to survive GRF cluster-size correction and was significant only at $p<0.001$, uncorrected).

Functional connectivity and cognitive performance

There were no significant correlations (after partialing out age) between functional connectivity and WM accuracy or RT for any of the ROIs where we had identified age-related differences in functional connectivity based on the time series regressor. However, because younger adults showed ceiling effects in performance on the WM task, we further explored correlations between prefrontal connectivity and WM performance in older adults only. Here, we found a trend for a negative association between right DLPFC connectivity to the right inferior parietal lobe (BA $40)$ and WM accuracy $(x=60, y=-30, z=34 ; r=-0.44, p=$ 0.07 ), and a positive association between MPFC connectivity to the right inferior frontal gyrus and WM accuracy $(x=56, y=10$, $z=16 ; r=0.54, p<0.05)$. There was no evidence for correlations of connectivity to RT.

\section{PET data and associations with functional connectivity}

As has been previously reported (Bäckman et al., 2011; Rieckmann et al., 2011), D1 receptor density in caudate was reduced by $\sim 20 \%$ in older compared to younger participants (mean $\mathrm{BP}_{\text {younger }}=1.71, \mathrm{SD}=0.28$; mean $\mathrm{BP}_{\text {old }}=1.33, \mathrm{SD}=0.23$, $\left.t_{(35)}=4.44, p<0.001\right)$.

We found that individual differences in D1 receptor density were positively associated with individual differences in functional connectivity (estimated from the time series regressor) of the right DLPFC to the right inferior parietal lobe (BA 40), as well as to midbrain after controlling for age (see Table 1 for coordinates and Fig. $2 A, B$ for scatter plots). Although we did not have any a priori hypotheses about midbrain connectivity, these results may be expected given the role of midbrain in DA synthesis and its DAergic connections to both caudate and right DLPFC (for review, see Haber, 2010). For MPFC connectivity, individual differences in D1 receptor density were negatively associated with individual differences in functional connectivity of the MPFC to right postcentral gyrus and right intraparietal sulcus (BA 40) after controlling for age (see Table 1 for coordinates and Fig. 2C,D for scatter plots). Because there were significant differences in accuracy on the WM task between age groups, we further controlled the partial correlation analyses for WM accuracy. The partial correlations between D1 receptor densities and functional connectivity estimates in all ROIs were not affected by controlling for accuracy, indicating that individual differences in functional connectivity were not merely a reflection of individual differences in task accuracy. Last, linear regression analyses showed no significant age $\times$ D1 interaction for any of the ROIs, indicating that the associations between D1 receptors and functional connectivity generalized across both age groups (see Fig. 2 for scatter plots across and within age groups).

Individual differences in caudate D1 receptor density were not associated with connectivity differences in the other ROIs, after controlling for age (e.g., in the ROIs where we found increased frontal bilaterality in older adults or increased connectivity between MPFC and PCC in younger adults).

\section{Discussion}

We assessed the association between caudate D1 receptor density and functional connectivity of the frontoparietal WM network and the DMN during performance of a WM task in younger and older adults. Several key findings emerged: (1) We replicated findings of reduced positive frontoparietal connectivity (Andrews-Hanna et al., 2007) and reduced negative connectivity between MPFC and the frontoparietal WM network (Sambataro et al., 2010) in older adults. (2) We also replicated previous reports of an age-related reduction in anterior-posterior coupling within the DMN (e.g., Andrews-Hanna et al., 2007; Grady et al., 2010; Sambataro et al., 2010). (3) We further found that older adults exhibited greater bilaterality of connectivity within the PFC. This was true both for the right DLPFC seed to medial and left prefrontal areas, and the MPFC seed to right prefrontal areas. These findings resemble previous data by Grady et al. (2010), although these authors also found increased frontoparietal coupling in older adults. (4) Most importantly, we derived estimates of caudate D1 receptor density using PET and the radioligand $\left[{ }^{11} \mathrm{C}\right] \mathrm{SCH} 23390$, and showed an association between D1 receptor density and functional connectivity of right DLPFC to right 

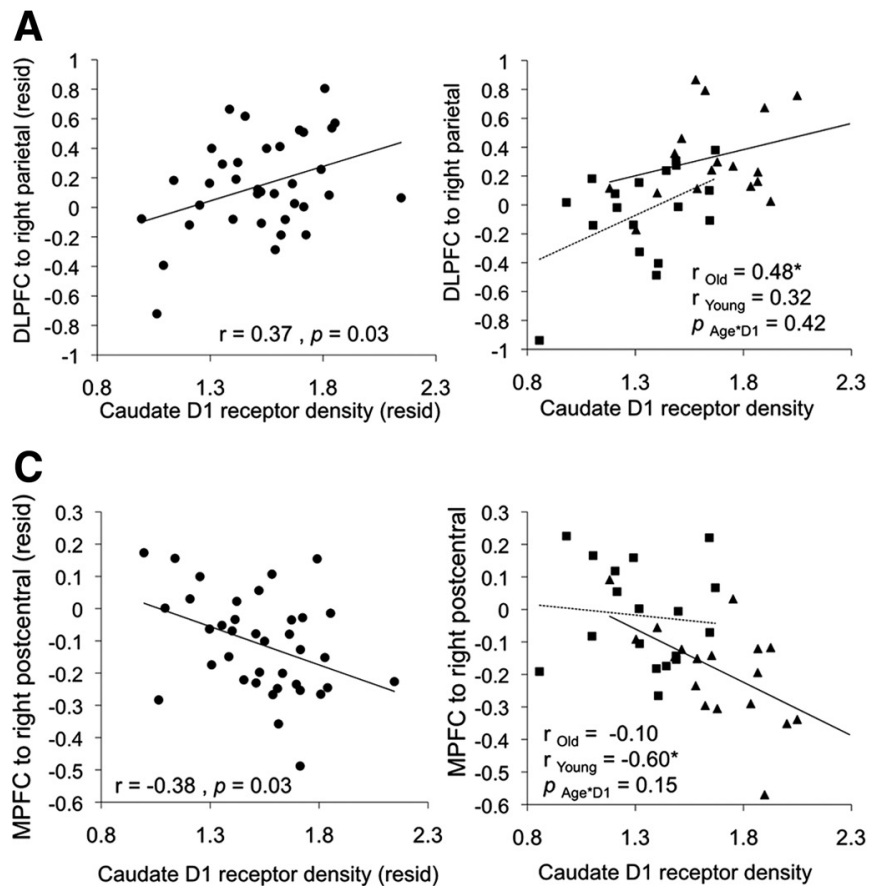

B
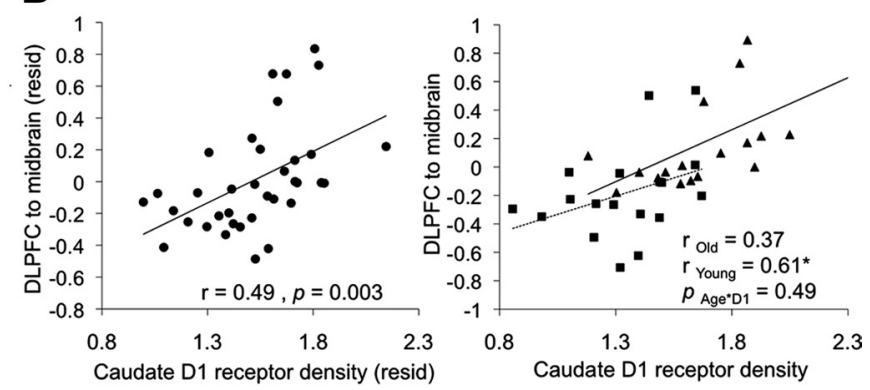

D
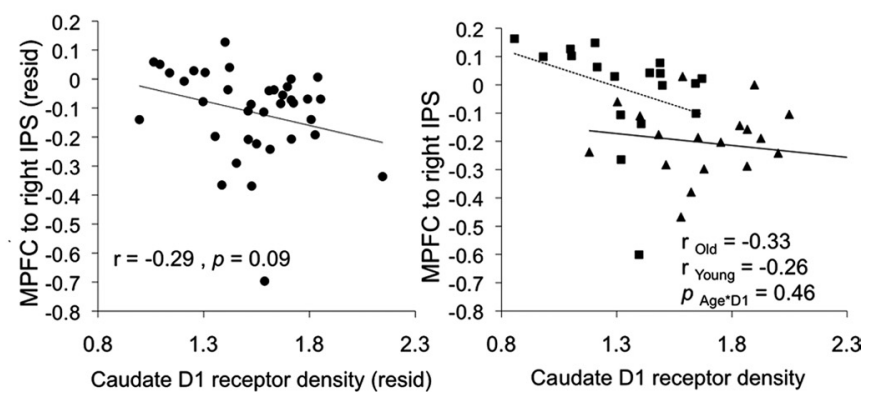

Figure 2. Scatter plots for correlations between D1 receptor density and functional connectivity estimates across the whole sample, after controlling for age (left), and by age group (right) for right DLPFC seed to right inferior parietal lobe (BA 40; $x=60, y=-30, z=34)(\boldsymbol{A})$, right DLPFC seed to midbrain $(x=8, y=-28, z=-10 ; 1$ young male outlier removed) (B), MPFC seed to right postcentral gyrus $(x=50, y=-22, z=38)(C)$, and MPFC seed to right intraparietal sulcus (IPS; $x=28, y=-46, z=48 ; 1$ young male outlier removed) (D). $r$ indicates the strength of the correlations ( ${ }^{*}$ significant at $p<0.05$ ) and $p_{\text {Age } \times \text { D1 }}$ the significance level of an age $\times D 1$ interaction. Squares represent older adults, and triangles represent younger adults. resid, Residuals (+mean).

parietal cortex (BA 40) and of MPFC to right intraparietal sulcus (BA 40) and postcentral gyrus, after controlling for age. Correlations between D1 receptor density and connectivity within the DMN or within the PFC did not survive statistical control for age. (5) Finally, we observed age-related reductions in connectivity between right DLPFC and midbrain, and a significant relationship between caudate D1 receptor density and DLPFC-midbrain connectivity after controlling for age. Although we did not have any hypotheses about midbrain connectivity, one might expect a relation of midbrain and DLPFC activity to caudate D1 receptors, as DA is synthesized in midbrain (ventral tegmental area and substantia nigra) and innervates the striatum and cortical areas (e.g., Haber, 2010). However, accurate midbrain imaging using MRI likely requires higher-resolution scans than were used in the present study (e.g., Düzel et al., 2009). We therefore consider the findings regarding DLPFC-midbrain connectivity as preliminary.

\section{Decreased frontoparietal connectivity in aging and links to caudate D1 receptor density}

Our results show that individual differences in caudate D1 receptor density are associated with individual differences in right DLPFC connectivity to right parietal cortex (BA 40) and MPFC "disconnectivity" to the intraparietal sulcus (BA 40) and postcentral gyrus during performance of a WM task after controlling for age (see Fig. 2). Consistent with this view, animal research has identified "open" cortico-striato-thalamo-cortical loops by which information is transferred between different cortical areas (Joel and Weiner, 2000). One such loop connects the associative areas of the PFC via the caudate to posterior sensorymotor areas/anterior parietal areas (Joel and Weiner, 1994). This pathway may provide a neurobiological underpinning of our finding that caudate D1 receptor functions are linked to func- tional connectivity between prefrontal and parietal areas (BA 40 and postcentral gyrus). The cross-sectional nature of our study does not allow causal inferences and cannot speak to whether DA changes across the lifespan are associated with lifespan changes in frontoparietal connectivity. However, consistent with the present study, age-related decreases in DA functions have been repeatedly shown in human and animal research (for review, see Bäckman et al., 2006, 2010). It is therefore biologically plausible that age-related reductions in caudate D1 receptor densities contribute to age-related reductions in functional connectivity of the prefrontal cortex to the right parietal cortex during WM. Further support for this notion comes from a recent study that found a relation between DA and age-related connectivity reductions of right inferior frontal gyrus to caudate during WM (Klostermann et al., 2011). Klostermann et al. constrained their fMRI analysis to the caudate, whereas in the present study, we used GRF cluster-size correction, which likely prevented us from detecting connectivity differences of smaller extent such as those with the striatum. However, together, the study by Klostermann et al. and the present findings converge to suggest that age-related connectivity differences of corticostriato-cortical pathways are linked to age-related differences in DA functions. These findings support the idea of a multilevel "cascade" model of cognitive aging (Buckner, 2004; Hedden and Gabrieli, 2004), where white matter losses (O'Sullivan et al., 2001; Andrews-Hanna et al., 2007), gray matter losses (Dickerson et al., 2008; Kalpouzos et al., 2011), amyloid accumulation (Hedden et al., 2009), and losses in the DA system may all contribute to age-related cognitive decline.

\section{Increased bilaterality in frontal connectivity in aging}

In addition to age-related decreases in frontoparietal connectivity, and their associations with D1 receptor density, we observed 
that older adults showed increased coupling of the DLPFC to MPFC and left inferior frontal gyrus, and of the MPFC to right inferior frontal gyrus, PFC, and anterior insula. Increased connectivity within the PFC for older adults was also found during the higher versus lower load condition, as suggested by the results from the PPI. Moreover, greater connectivity within the PFC (MPFC to inferior frontal gyrus) was related to better performance in older adults, whereas connectivity of DLPFC with right parietal cortex was related to worse performance. Grady et al. (2010) also found a positive association between greater prefrontal connectivity and cognitive task performance in older adults, and it has been suggested that increasing recruitment of the frontal lobes in older adults may reflect neural reorganization and compensation (e.g., Cabeza, 2002; Grady et al., 2010). Such an interpretation would also fit our observation that greater bilateral connectivity within the PFC was related to reduced frontoparietal connectivity of the DLPFC. Moreover, the fact that we found associations between D1 receptor densities and connectivity only for the frontoparietal connections but not for the frontofrontal connections suggests that different neurobiological processes underlie these pathways. Together, direct corticocortical pathways within the frontal cortex may parallel indirect cortico-striatothalamo-cortical pathways and be able to compensate for DArelated connectivity losses in the indirect pathway. However, recent longitudinal fMRI evidence has shown that cross-sectional frontal overactivation in older adults may be the result of biased sampling toward high-functioning older adults (Nyberg et al., 2010). Nyberg et al. found that, although a cross-sectional analysis of task-based fMRI data suggested age-related increases in frontal activation, longitudinal analysis clearly showed decreases of frontal activation within individuals over 6 years. These results underscore the need for longitudinal work on DA functions and connectivity to clarify the role of DA in age-related changes in frontoparietal as well as bilateral-frontal connectivity.

\section{Limitations of the study}

We found no significant correlations of D1 receptor densities to WM accuracy or RT either across both groups (partialing out age) or within groups. The absence of correlations runs counter to previous findings linking striatal DA to WM performance (Landau et al., 2009; Wallace et al., 2011). Conceivably, the absence of correlations reflects limitations of the task used in the current study. Younger adults performed at ceiling and older adults also had no difficulties performing the task, which likely led to insufficient range in performance to detect reliable effects. Ceiling effects in the young are likely also the reason that we failed to observe interactions between task load and functional connectivity in the PPI analyses in this age group.

That said, the fact that we found significant correlations between functional connectivity estimates and D1 receptor densities, but not between cognitive performance and D1 receptor densities, is consistent with previous studies that have found functional connectivity to be a more sensitive estimate of underlying neurobiological processes than neuropsychological performance (e.g., Au Duong et al., 2005; Boorman et al., 2007; Hedden et al., 2009).

A further limitation of the present study is that we only assessed D1 receptor densities. We used a ligand for the D1 receptor subtype because of its significance for WM (e.g., Cools and D'Esposito, 2010) and its relationship to the BOLD response (Knutson and Gibbs, 2007). Yet, several studies have also implicated the D2 receptor subtype, as well as the DA transporter and DA synthesis capacity, in age-related cognitive changes (for re- view, see Bäckman et al., 2010) and, most interestingly, in alterations of the DMN (e.g., Nagano-Saito et al., 2009; Braskie et al., 2011), where we failed to observe relationships with D1 receptor density. The relative importance of D1 or D2 receptors and other markers of the DA system to age-related changes in functional connectivity in task-related cortical networks remains to be determined.

\section{Conclusions}

This study shows that individual differences in frontoparietal coupling during WM performance are associated with individual differences in caudate D1 receptor density. These results suggest that age-related changes in D1 receptor densities may contribute to age-related reductions in functional connectivity of the prefrontal and parietal cortices. The data are consistent with findings that striatal DA receptors modulate functioning of corticostriato-cortical loops that transfer information between prefrontal and parietal areas.

\section{References}

Andrews-Hanna JR, Snyder AZ, Vincent JL, Lustig C, Head D, Raichle ME, Buckner RL (2007) Disruption of large-scale brain systems in advanced aging. Neuron 56:924-935.

Au Duong M-V, Audoin B, Boulanouar K, Ibarrola D, Malikova I, ConfortGouny S, Celsis P, Pelletier J, Cozzone PJ, Ranjeva J-P (2005) Altered functional connectivity related to white matter changes inside the working memory network at the very early stages of MS. J Cereb Blood Flow Metab 25:1245-1253.

Bäckman L, Small B, Wahlin $\AA$ (2001) Aging and memory: cognitive and biological perspectives. In: Handbook of the psychology of aging, Ed 5 (Birren J, Schaie K, eds), pp 349-377. San Diego: Academic.

Bäckman L, Nyberg L, Lindenberger U, Li S-C, Farde L (2006) The correlative triad among aging, dopamine, and cognition: current status and future prospects. Neurosci Biobehav Rev 30:791-807.

Bäckman L, Lindenberger U, Li S-C, Nyberg L (2010) Linking cognitive aging to alterations in dopamine neurotransmitter functioning: recent data and future avenues. Neurosci Biobehav Rev 34:670-677.

Bäckman L, Karlsson S, Fischer H, Karlsson P, Brehmer Y, Rieckmann A, MacDonald SW, Farde L, Nyberg L (2011) Dopamine D1 receptors and age differences in brain activation during working memory. Neurobiol Aging 32:1849-1856.

Baddeley A (2003) Working memory: looking back and looking forward. Nat Rev Neurosci 4:829-839.

Boorman ED, O'Shea J, Sebastian C, Rushworth MFS, Johansen-Berg H (2007) Individual differences in white-matter microstructure reflect variation in functional connectivity during choice. Curr Biol 17:1426-1431.

Braskie MN, Landau SM, Wilcox CE, Taylor SD, O’Neil JPO, Baker SL, Madison CM, Jagust WJ (2011) Correlations of striatal dopamine synthesis with default network deactivations during working memory in younger adults. Hum Brain Mapp 32:947-961.

Buckner RL (2004) Memory and executive function in aging and AD: multiple factors that cause decline and reserve factors that compensate. Neuron 44:195-208.

Buckner RL, Andrews-Hanna JR, Schacter DL (2008) The brain's default network anatomy, function and relevance to disease. Ann N Y Acad Sci 1124:1-38.

Cabeza R (2002) Hemispheric asymmetry reduction in older adults: the HAROLD model. Psychol Aging 17:85-100.

Callicott JH, Mattay VS, Bertolino A, Finn K, Coppola R, Frank JA, Goldberg TE, Weinberger DR (1999) Physiological characteristics of capacity constraints in working memory as revealed by functional MRI. Cereb Cortex 9:20-26.

Cools R, D’Esposito M (2010) Dopaminergic modulation of flexible cognitive control in humans. In: Dopamine handbook (Iversen L, Iversen S, Dunnett S, Björklund A, eds), pp 38-48. New York: Oxford UP.

Curtis CE, D'Esposito M (2003) Persistent activity in the prefrontal cortex during working memory. Trends Cogn Sci 7:415-423.

Dickerson BC, Fenstermacher E, Salat DH, Wolk DA, Maguire RP, Desikan R, Pacheco J, Quinn BT, Van der Kouwe A, Greve DN, Blacker D, Albert MS, 
Killiany RJ, Fischl B (2008) Detection of cortical thickness correlates of cognitive performance: Reliability across MRI scan sessions, scanners, and filed strengths. Neuroimage 39:10-18.

Düzel E, Bunzeck N, Guitart-Masip M, Wittmann B, Schott BH, Tobler PN (2009) Functional imaging of the human dopaminergic midbrain. Trends Neurosci 32:321-328.

Fischer H, Nyberg L, Karlsson S, Karlsson P, Brehmer Y, Rieckmann A, MacDonald SWS, Farde L, Bäckman L (2010) Simulating neurocognitive aging: Effects of a dopaminergic antagonist on brain activity during working memory. Biological Psychiatry 67:575-580.

Fox MD, Snyder AZ, Vincent JL, Corbetta M, Van Essen DC, Raichle ME (2005) The human brain is intrinsically organized into dynamic, anticorrelated functional networks. Proc Natl Acad Sci U S A 102:9673-9678.

Grady CL, Protzner AB, Kovacevic N, Strother SC, Afshin-Pour B, Wojtowicz M, Anderson JA, Churchill N, McIntosh AR (2010) A multivariate analysis of age-related differences in default mode and task-positive networks across multiple cognitive domains. Cereb Cortex 20:1432-1447.

Grahn JA, Parkinson JA, Owen AM (2008) The cognitive functions of the caudate nucleus. Prog Neurobiol 86:141-155.

Haber S (2010) Convergence of limbic, cognitive, and motor cortico-striatal circuits within dopamine pathways in primate brain. In: Dopamine handbook (Iversen L, Iversen S, Dunnett S, Björklund A, eds), pp 38-48. New York: Oxford UP.

Hall H, Farde L, Sedvall G (1988) Human dopamine receptor subtypes-in vitro binding analysis using 3H-SCH 23390 and $3 \mathrm{H}$-raclopride. J Neural Transm 73:7-21.

Halldin C, Stone-Elander S, Farde L, Ehrin E, Fasth KJ, Långström B, Sedvall G (1986) Preparation of 11C-labeled SCH 23390 for the in vivo study of dopamine D1 receptors using positron emission tomography. Int J Rad Appl Instrum A 37:1039-1043.

Hampson M, Driesen N, Roth JK, Gore JC, Constable RT (2010) Functional connectivity between task-positive and task-negative brain areas and its relation to working memory performance. Magn Reson Imaging 28:1051-1057.

Hedden T, Gabrieli JDE (2004) Insights into the aging mind: A view from cognitive neuroscience. Nat Rev Neurosci 5:87-96.

Hedden T, Van Dijk KRA, Becker JA, Mehta A, Sperling RA, Johnson KA, Buckner RL (2009) Disruption of functional connectivity in clinically normal older adults harboring amyloid burden. J Neurosci 29:12686-12694.

Innis RB, Cunningham VJ, Delforge J, Fujita M, Gjedde A, Gunn RN, Holden J, Houle S, Huang SC, Ichise M, Iida H, Ito H, Kimura Y, Koeppe RA, Knudsen GM, Knuuti J, Lammertsma AA, Laruelle M, Logan J, Maguire RP, et al. (2007) Consensus nomenclature for in vivo imaging of reversible binding radioligands. J Cereb Blood Flow Metab 27:1533-1539.

Jenkinson M, Bannister P, Brady M, Smith S (2002) Improved optimization for the robust and accurate linear registration and motion correction of brain images. Neuroimage 17:825-841.

Joel D, Weiner I (1994) The organization of the basal ganglia-thalamocortical circuits: open interconnected rather than closed segregated. Neuroscience 63:363-379.

Joel D, Weiner I (2000) The connections of the dopaminergic system with the striatum in rats and primates: an analysis with respect to the functional and compartmental organization of the striatum. Neuroscience 96:451-474.

Kalpouzos G, Persson J, Nyberg L (2011) Local brain atrophy accounts for functional activity differences in normal aging. Neurobiol Aging. Advance online publication. Retrieved April 23, 2011. doi:10.1016/j.neurobiolaging. 2011.02.021.

Klostermann E, Braskie M, Landau S, O'Neil JP, Jagust WJ (2011) Dopamine and frontostriatal networks in cognitive aging. Neurobiol Aging. Advance online publication. Retrieved April 19, 2011. doi:10.1016/j.neurobiolaging. 2011.03.002.

Knutson B, Gibbs SE (2007) Linking nucleus accumbens dopamine and blood oxygenation. Psychopharmacology 191:813-822.

Lammertsma AA, Hume SP (1996) Simplified reference tissue model for PET studies. Neuroimage 4:153-158.

Landau SM, Lal R, O’Neil JP, Baker S, Jagust WJ (2009) Striatal dopamine and working memory. Cereb Cortex 19:445-454.

Lehéricy S, Ducros M, Van de Moortele P-F, Francois C, Thivard L, Poupon C, Swindale N, Ugurbil K, Kim D-S (2004) Diffusion tensor fiber tracking shows distinct corticostriatal circuits in humans. Ann Neurol 55:522-529.

Meltzer CC, Leal JP, Mayberg HS, Wagner HN Jr, Frost JJ (1990) Correction of PET data for partial volume effects in human cerebral cortex by MR imaging. J Comput Assist Tomogr 14:561-570.

Nagano-Saito A, Liu J, Doyon J, Dagher A (2009) Dopamine modulates default mode network deactivation in elderly individuals during the Tower of London task. Neurosci Lett 458:1-5.

Nagel IE, Preuschhof C, Li S-C, Nyberg L, Bäckman L, Lindenberger U, Heekeren HR (2011) Load modulation of BOLD response and connectivity predicts working memory performance in younger and older adults. J Cogn Neurosci 23:2030-2045.

Nyberg L, Andersson M, Forsgren L, Jakobsson-Mo S, Larsson A, Marklund P, Nilsson L-G, Riklund K, Bäckman L (2009) Striatal dopamine D2 binding is related to frontal BOLD response during updating of long-term memory representations. Neuroimage 46:1194-1199.

Nyberg L, Salami A, Andersson M, Eriksson J, Kalpouzos G, Kauppi K, Lind J, Pudas S, Persson J, Nilsson L-G (2010) Longitudinal evidence for diminished frontal cortex function in aging. Proc Natl Acad Sci U S A 107:22682-22686.

Rieckmann A, Karlsson S, Karlsson P, Brehmer Y, Fischer H, Farde L, Nyberg L, Bäckman L (2011) Dopamine D1 receptor associations within and between dopaminergic pathways in young and elderly adults: links to cognitive performance. Cereb Cortex 21:2023-2032.

Roland PE, Graufelds CJ, Wåhlin J, Ingelman L, Andersson M, Ledberg A, Pedersen J, Åkerman S, Dabringhaus A, Zilles K (1994) Human brain atlas for high resolution functional and anatomical mapping. Hum Brain Mapp 1:173-184.

Sambataro F, Murty VP, Callicott JH, Tan H-Y, Das S, Weinberger DR, Mattay VS (2010) Age-related alterations in default mode network: Impact on working memory performance. Neurobiol Aging 31:839-852.

Schott BH, Minuzzi L, Krebs RM, Elmenhorst D, Lang M, Winz OH, Seidenbecher CI, Coenen HH, Heinze H-J, Zilles K, Düzel E, Bauer A (2008) Mesolimbic functional magnetic resonance imaging activations during reward anticipation correlate with reward-related ventral striatal dopamine release. J Neurosci 28:14311-14319.

Smith SM, Jenkinson M, Woolrich MW, Beckmann CF, Behrens TE, Johansen-Berg H, Bannister PR, De Luca M, Drobnjak I, Flitney DE, Niazy RK, Saunders J, Vickers J, Zhang Y, De Stefano N, Brady JM, Matthews PM (2004) Advances in functional and structural MR image analysis and implementation as FSL. Neuroimage 23 [Suppl 1]:S208-S219.

Toro R, Fox PT, Paus T (2008) Functional coactivation map of the human brain. Cereb Cortex 18:2553-2559.

Wallace DL, Vytlacil JJ, Nomura EM, Gibbs SE, D'Esposito M (2011) The dopamine agonist bromocriptine differentially affects fronto-striatal functional connectivity during working memory. Front Hum Neurosci 5:32.

Ystad M, Hodneland E, Adolfsdottir S, Haász J, Lundervold AJ, Eichele T, Lundervold A (2011) Cortico-striatal connectivity and cognition in normal aging: a combined DTI and resting state fMRI study. Neuroimage $55: 24-31$. 